\title{
Repair of Electronics for Long Duration Spaceflight
}

\author{
Richard D. Pettegrew ${ }^{1}$ and John Easton ${ }^{1}$ \\ National Center for Space Exploration Research, Cleveland, OH 44135 \\ and \\ Peter Struk ${ }^{2}$ \\ NASA Glenn Research Center, Cleveland, OH 44135
}

[Abstract] To reduce mission risk, long duration spaceflight and exploration activities will require greater degrees of self-sufficiency with regards to repair capability than have ever been employed before in space exploration. The current repair paradigm of replacing Orbital Replacement Units (ORUs) of malfunctioning avionics and electronic hardware will be impractical, since carrying all of the spares that could possibly be needed for a long duration mission would require upmass and volume at unprecedented and unacceptable levels. A strategy of component-level repair for electronics, however, could significantly reduce the mass and volume necessary for spares and enhance mission safety via a generic contingency capability. This approach is already used to varying degrees by the U.S. Navy, where vessels at sea experience some similar constraints such as the need for self sufficiency for moderately long time periods, and restrictions on volume of repair spares and infrastructure.

The concept of conducting component-level repairs of electronics in spacecraft requires the development of design guidelines for future avionics (to enable repair), development of diagnostic techniques to allow an astronaut to pinpoint the faulty component aboard a vastly complex vehicle, and development of tools and methodologies for dealing with the physical processes of replacing the component. This physical process includes tasks such as conformal coating removal and replacement, component removal, replacement, and alignment--all in the difficulty of a reduced gravity environment. Further, the gravitational effects on the soldering process must be characterized and accounted for to ensure reliability of the newly repaired components.

The Component-Level Electronics-Assembly Repair (CLEAR) project under the NASA Supportability program was established to develop and demonstrate the practicality of this repair approach. CLEAR involves collaborative efforts between NASA's Glenn Research Center, Langley Research Center, Johnson Space Center, the National Center for Space Exploration Research, and the U.S. Navy. The project goals are 1) develop and demonstrate a manually-operated electronics repair capability to be conducted in a spacecraft environment; and 2) develop guidelines for designs of electronics that facilitates component-level repair for future space exploration efforts. This multi-faceted program utilizes a cross-disciplinary approach to examine pre- and post-repair diagnostics, conformal coating removal and replacement, component soldering, and electronics design for supportability. These areas are investigated by a combination of trade studies, ground based testing, reduced gravity aircraft testing, and actual spaceflight testing on the International Space Station (ISS) in multiple experiments. This paper details the efforts of this program, with emphasis on early trade study results, ground-based efforts, and two upcoming ISS experiments.

\section{Introduction}

THE historical approach to electronics repair on manned spaceflight missions (particularly those aboard the International Space Station \{ISS\}) has been to replace modular subassemblies called Orbital Replacement Units (ORU's). The astronaut simply replaces the malfunctioning ORU with a spare, and then returns the faulty ORU to Earth, where it is diagnosed. The unit may then be repaired and returned to a pool of spares awaiting re-use in space ${ }^{1}$. This approach allows for simplicity in the diagnosis of the problem, as the fault only has to be isolated to a given ORU, rather than determining the fault down to the component level. Crew training and experience in performing repairs are minimized, and there is a high degree of confidence in the repair, as the ORU can be tested extensively on the ground prior to being re-launched to the station.

\footnotetext{
${ }^{1}$ Staff Scientist, 21000 Brookpark Rd, Mail Stop 110-3, Cleveland, Ohio, 44135, AIAA Member.

${ }^{2}$ Aerospace Engineer, 21000 Brookpark Rd, Mail Stop 110-3, Cleveland, Ohio, 44135, AIAA Member.
} 
Conversely, this approach carries with it a severe penalty in terms of the logistics support required. Conceptually, it is easy to imagine the penalty in mass and volume that is levied when an entire ORU (which may weigh $\sim 25$ lbs or more) is launched to remedy a failure of a single, small component such as a resistor, transistor, or other electrical component. While this approach is expensive, it may be acceptable for low earth orbit (LEO) missions if they are re-supplied regularly since large quantities of spares would not need to be stored on orbit.

Designers of future long-duration missions (such as NASA's program for manned exploration of the Moon and Mars) will not have the option of regularly scheduled re-supply flights to mitigate the mission's logistical needs. Under the current repair strategy of ORU replacement, mission planners and spacecraft designers will be forced to choose one of several scenarios:

1) Over sizing the spacecraft to accommodate the large number of spare ORU's needed for proper safety margin, resulting in much greater vehicle size and costs.

2) Placing severe limits on the number of spares that are carried, resulting in increased risk to crew and mission.

3) Pre-position stores of spares, either at the destination, or at some designated 'way point'. This approach will require the mission planners to assume the types and number of ORU's that should be pre-positioned, and also will entail greater program cost.

An alternative to these scenarios is to implement a component-level repair strategy. This could serve to greatly reduce the planned-spare ORU requirements, while providing a capability to handle unforeseen repair contingencies. The need to handle such unexpected events has been illustrated by several events in NASA flight history. The successful conclusion of the Apollo 13 mission, after an explosion severely damaged the service module, highlights the benefits of having the capability to implement on-the-spot repairs. The consequence of failing to allow for offnominal repairs was demonstrated in the tragic loss of the shuttle Columbia; even if the crew had been aware of the problems with the leading edge tiles, they were not equipped to make repairs in-flight. Since that incident, NASA has made considerable efforts to allow for contingency repairs of problems of that nature ${ }^{2}$.

NASA is also now in the planning stages for an ambitious expansion of their exploration efforts, including longduration missions to the Moon and (eventually) Mars. In an effort to address the conflicting needs of minimizing volume and mass, while maximizing mission safety and reliability, research efforts are underway aimed at developing a component-level electronic repair capability for use on such missions. This task, named 'CLEAR' (Component-Level Electronic-Assembly Repair), encompasses a combination of laboratory testing as well as reduced gravity testing aboard NASA's reduced gravity aircraft, and flight testing aboard the International Space Station. This paper details recent work and upcoming plans for the CLEAR team's efforts in this area.

\section{Past Work}

CLEAR, which started in early 2006, traces its roots back to the Soldering in Reduced Gravity Experiment (SoRGE) that was conducted jointly by researchers from NASA's Glenn Research Center, Johnson Spaceflight Center, and the National Center for Space Exploration Research, beginning in 2000 $0^{3,4,5,6,7}$. The SoRGE effort examined the effects of reduced gravity on the soldering process aboard NASA's Reduced-Gravity Aircraft (both KC-135 and DC-9). Other limited studies have also been performed on different aspects of soldering in reduced gravity 8 .

SoRGE examined a plated-through-hole (PTH) joint configuration as its primary configuration (a limited number of surface mount, dual-flat-pack samples were also formed). All joints were formed manually using a soldering iron and rosin-core, 60/40 $(\mathrm{Sn} / \mathrm{Pb})$ solder, similar to that found in a soldering kit currently on board the ISS ${ }^{1}$. Joints were formed in various reduced-gravity environments ${ }^{10,11}$ including (nominally) Martian, Lunar, and zero gravity levels ${ }^{*}$. Samples were then subjected to an external visual inspection (using NASA standards ${ }^{12}$ ) and destructive internal examination. This internal examination was performed by mounting the solder joint in a metallographic mount, then grinding the joint down to a mid-plane (Figure 1). The sectioned joint was then imaged, and internal void volume fractions were calculated $\mathrm{d}^{3,4,6,7}$.

Results showed approximately three times the void fraction in joints formed in (nominally) zero gravity as those formed in normal gravity, 3,5,7. Further, the void fraction increased linearly (within the resolution limits of the experiment) as gravity level was decreased below approximately Martian gravity levels. Changes in joint geometry were also observed, with the reduced-gravity joints being more symmetric.

\footnotetext{
* Brief periods ( 25 seconds) of reduced-gravity are experienced aboard these aircraft as they fly parabolic trajectories which are calculated to provide the desired relative acceleration levels.
} 
The increase in void fraction was attributed to two possible factors. Entrapped (vaporized) flux within the solder may solidify within the joints, due to the lack of buoyant forces which tend to drive out such vapors in normal gravity. Also, evolved water vapor from the circuit card itself may contribute to the void fraction.

These hypotheses were tested using two separate strategies to mitigate and isolate those possible vapor sources. In the first case, a solid-core solder (i.e., with no flux core) was used in conjunction with an externally applied liquid flux. The joint was preheated for several seconds (before adding solder) to allow the flux to activate and vaporize, thereby reducing the amount of flux available to be trapped in the joint. The second strategy involved desiccating the boards, prior to soldering. This was done by baking the boards in a low-heat drying oven for several hours prior to soldering. Both strategies were applied independently, so that changes in void fraction could be attributed correctly. Both attempts at void fraction mitigation proved effective. In both cases, the void fraction was reduced significantly, approaching the levels observed in normal gravity, with the original technique ${ }^{3,4,5,7}$.

The SoRGE effort is believed to be the first systematic study of the effects of reduced gravity (including partial gravity) on the soldering process. These results demonstrate the effects that the acceleration environment can have, and underscore the need to understand how this unique environment can affect the process of component-level electronics repair. The continuation of these efforts, and the need to verify the results in a true microgravity environment, led to the development of an SDTO (Station Development Test Objective) flight experiment ${ }^{13}$ that has been incorporated into the CLEAR project, discussed next.

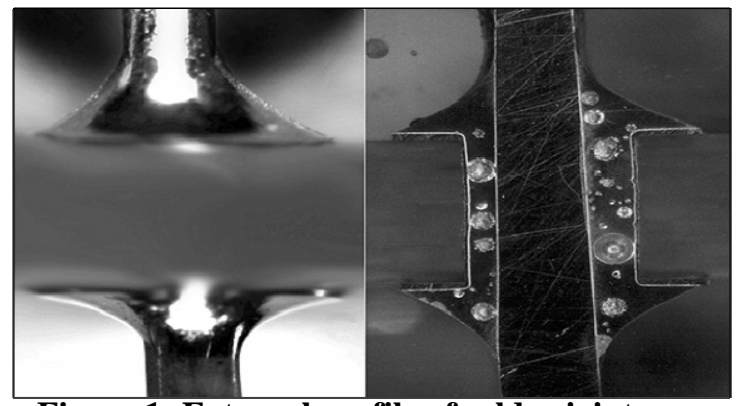

Figure 1: External profile of solder joint (left), and cross section of joint (right) showing internal voids.

\section{The CLEAR Project}

The CLEAR project differs from the previous SoRGE project through the scope of the efforts. SoRGE was intended to examine how reduced gravity affects the soldering process itself, particularly with respect to void fraction. The scope of CLEAR is much broader, encompassing the end-to-end process of repairing failed electronics at the component level. This end-to-end process entails diagnosis of what element has failed on a given board, removal of the conformal coating (and subsequent replacement of the coating after the repair is completed), removal of the failed component and board cleanup, and placement, alignment and soldering of the new component. Further, the repair must be verified before the board is returned to service.

Within the scope of the end-to-end repair process, the goal of the CLEAR project is to raise the Technology Readiness Level (TRL) ${ }^{14}$ of component-level electronic repair, as a repair strategy, to a level of TRL- $6{ }^{* *}$. The CLEAR project involves a range of activities, including trade studies, ground-based laboratory research (both normal gravity and reduced gravity), and flight activities aboard the ISS. This approach provides a cost effective way of developing the technology and raising the TRL level, by demonstrating only the gravity-dependent portions of the repair processes in a flight environment, while demonstrating other elements that are unaffected by gravity using ground-based methods.

\section{A. Trade Study}

CLEAR's initial efforts centered on a comprehensive trade study ${ }^{1}$, which was completed in the fall of 2006. This trade study examined the current state of electronics repair technology, with the perspective of the applicability of those technologies for use in a spaceflight environment. A key element of the trade study was a series of case studies examining how electronic repairs are performed in three different settings, including how the design philosophy for electronics used in each of these settings affects the repair process. The three settings were NASA's current repair strategy for ground-based repair of electronics, the U.S. Navy's repair program (for ships at sea, and at the shore-based depot level), and a commercial electronics fabrication company.

\footnotetext{
** The TRL is a scale that NASA uses to rate the maturity level of a given technology, ranging from 1 (where basic principles have been observed and reported) to 9 ("mission proven" through successful use on spaceflight missions). A TRL level of 6 indicates that the technology has been tested in a relevant, end-to-end environment.
} 
The results of these case studies demonstrate several key points:

1. It is important that the electronics are designed so that they are amenable to 'field' repair.

2. The diagnostics used to determine faulty components on a board are often fairly large and complex, and must be simplified for use in flight. The Navy addresses this (on ships at sea) using non-functional testers (equipment which tests the board in an unpowered state) and a comprehensive database to compare the electronic signature of the faulty board, to a known 'good' standard.

3. It is important for NASA to maintain design information and other (often proprietary) knowledge such as embedded source code, particularly on components or systems that are developed by outside vendors. This knowledge is crucial in developing and implementing component-level repair strategies.

4. The level to which component-level repair can be implemented will be a function of training of the crew, the tools and infrastructure provided, and the degree to which the electronics systems were designed for repair.

The balance of the trade study examined current repair technologies, and evaluated them for their applicability to a spaceflight mission, and provided recommendations as to which of these technologies are most amenable to this application, as well as design recommendations based on the case studies.

\section{B. Ground-Based Experimental Efforts}

CLEAR's ground-based experimental work focuses on several areas. First, continuing efforts will be made towards understanding the role that gravity plays in the formation of solder joints. This research, which is an extension of the work started with the SoRGE project, will again be conducted aboard NASA's Reduced Gravity Aircraft (Figure 2), which is classified as a ground-based research facility. The relatively low cost of aircraft experiments (compared to the cost of spaceflight experiments) means that fairly large sample populations can be developed, and multiple configurations and techniques can be tested in an affordable way. In addition to testing different configurations, other methods for void fraction mitigation, such as the use of different solder alloys and flux compositions, will also be tested and evaluated.

While the airplane provides a good platform on which to perform large numbers of brief tests (such as soldering a single joint), the duration of a single parabola is too short to allow high-fidelity testing of the end-to-end repair process. Further, though good initial results can be obtained on the airplane, true microgravity results will only be obtained using spacecraft experiments, due to the residual accelerations (termed 'g-jitter') experienced aboard the plane ${ }^{* * *}$. Spaceflight experiments are also a part of the CLEAR team's plan, and are discussed in a later section.

While the reduced-gravity testing on the aircraft will provide important information on the soldering process itself, a parallel effort will take place to develop and demonstrate the techniques and hardware needed to diagnose what component has failed on a board. Since no gravitational effects are expected on the diagnostic process, this effort can take place in a normal-gravity, benchtop environment.

Work has already begun on the design of a prototype, benchtop repair demonstrator system, which is scheduled for completion in 2008. This system will allow demonstration of all aspects of the end-to-end repair process including diagnostics, which is the primary emphasis of this effort. This demonstration will feature an operator (with training and experience comparable to that of an astronaut) diagnosing a board that has an unknown, failed component installed on it. A database of the proper electronic signatures of the components on that board will be developed beforehand, giving the operator a reference to use in conjunction with a non-functional test system (similar to the Navy's strategy for ships at sea).

After successfully diagnosing which component is faulty, the operator will then complete all of the physical repair steps in the process. While the gravity-dependent, physical repair processes will need to be demonstrated in a

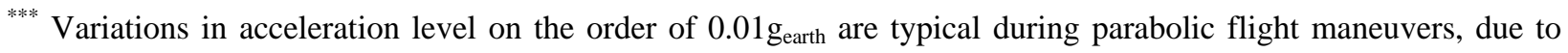
airframe vibrations, weather effects, etc.
} 
flight environment (described later), the demonstration of successful diagnosis of the component, using these techniques and equipment, will combine with those flight results to raise the TRL level of this repair strategy to level 6 .

\section{Spaceflight Experiments}

The previously mentioned ground-based research activities provide a cost-effective foundation for the development of a component-level repair capability to be used during long duration missions. While much insight can be gained from benchtop (normal gravity) and reduced-gravity aircraft operations, the final proof of the viability of component-level electronics repair for this application can only come using flight experiments in a spacecraft environment. Successful, in-space demonstration of the gravity dependent physical processes, combined with benchtop demonstration of the non-gravity dependent processes, will serve to fulfill the overall objective of raising the TRL to level 6.

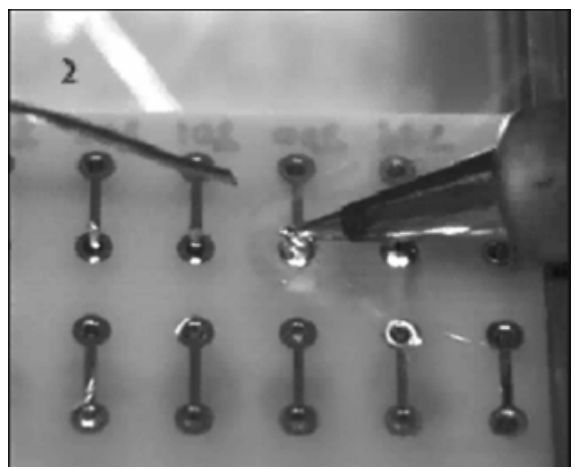

There are currently two flight experiments scheduled for the CLEAR project, as well as efforts to analyze samples produced during an actual, unplanned repair that was performed aboard the ISS. Both of the new experiments utilize the SDTO (Station Development Test Objective) program, which provides flight opportunities for engineering experiments that have direct application to either ISS or exploration. The CLEAR efforts fit this definition, as the ability to perform component-level electronics repair (and thereby reduce the logistical footprint required) is relevant and useful for any long duration efforts, be it either ISS or deep-space missions.

The first SDTO effort for the CLEAR project (designated SDTO17003-U by NASA) is a follow-up to SoRGE, and was initiated during the later stages of the SoRGE project. This experiment, launched

Figure 3: Test operator soldering a plated through-hole (PTH) joint. September 9, 2006 aboard the Shuttle Discovery, is currently onboard the ISS and will be performed in the near future. This effort will have the astronaut manually solder a series of plated through-hole joints (Figure 3), similar to the previous work done onboard the reduced gravity aircraft. The results from this experiment will be used to confirm and extend the results from the aircraft testing, which showed that the void fraction increased as the gravitation level decreased. Since the acceleration environment onboard the ISS is lower (and much more steady) than that experienced aboard the airplane, greater levels of void fraction are expected. This experiment will also give insight into the need and usefulness of certain tools in the spacecraft environment, and will allow assessment of the viability of remote video-training of the crew, which could be useful for contingency training of the crew if unexpected repair efforts are needed.

A follow-up to SDTO 17003-U is already in the planning and development stage, and may be ready to fly in the third quarter of 2007. This experiment will examine all aspects of the repair process (not just soldering), with the exception of the diagnostics. The concept of this effort is to demonstrate the viability of removing the conformal coating, safely removing the component from the board and cleaning the area (with no damage to the board traces), replacing and soldering the component, and reapplying a conformal coating. This process will be performed on a functional board (Figure 4) with different part configurations (PTH and surface mount devices) and different lead sizes, to give a realistic representation of the general types of repairs that could be required during a mission. After completion of the experiment, the boards will be returned to earth for analysis.

An additional effort is underway to analyze samples produced by Astronaut Don Pettit during an unplanned repair of the 'Arctic Freezer', a device used during Expedition 6 aboard the ISS to store science experiment samples. Using only tools and supplies that were

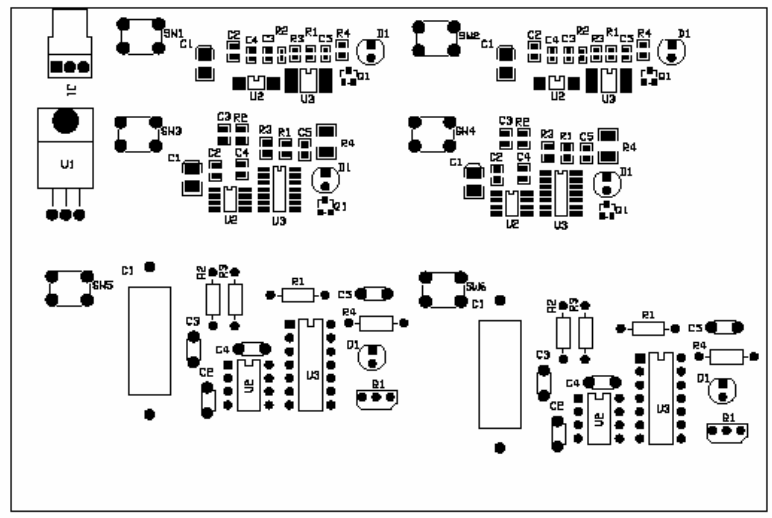

Figure 4: Schematic layout of test board for CLEAR's second SDTO experiment. 


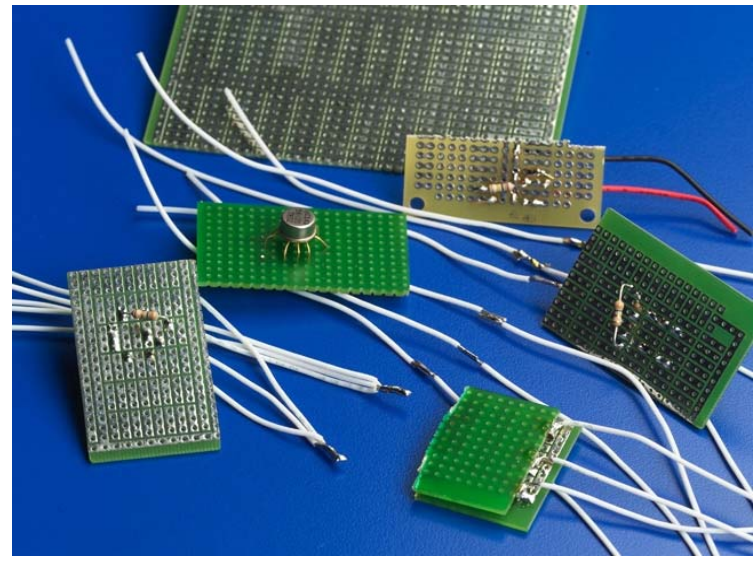

Figure 5: Representative samples (formed in normal gravity) used for baseline comparison with those produced by Astronaut Don Pettit during the Arctic Freezer repair. The repairs were conducted aboard ISS during Expedition 6.

already aboard the ISS, Pettit was able to restore functionality of the freezer using soldering techniques (Figure 5); these joints are currently undergoing analysis for void fraction content. Results from these samples are expected in early 2007, with findings to be published soon thereafter.

\section{Summary}

NASA's historical solution to the problem of inflight electronics repair has been the replacement of subunits called Orbital Replacement Units. This approach requires significant logistical support through regular resupply flights. Component-level repair of electronics is an option that can reduce the logistical footprint required to support long-duration space missions, as re-supply flights are likely to be few or non-existent.

Earlier work on the SoRGE project showed differences between solder joints produced in normal gravity and those produced in reduced gravity, including significant increase in void fraction, and changes in overall geometry. This work was the predecessor to the CLEAR project, which is an effort to develop and demonstrate component-level electronics repair, and raise the maturity level of this technology to TRL-6. CLEAR differs from the SoRGE effort in that CLEAR will examine all aspects of the repair process (including diagnosis of what component on the board has failed), while the scope of SoRGE was limited to the study of how the soldering process was affected by reduced gravity.

The CLEAR efforts include a comprehensive trade study, which was completed in the fall of 2006. This trade study included case studies of current NASA, U.S. Navy, and commercial repair practices, as well as an overview of the current state-of-the-art in electronics repair technologies. Both ground-based and flight-based experimental efforts have also been undertaken, including the development of a benchtop repair demonstration system (including diagnostics), reduced-gravity aircraft experiments, and SDTO flight activities aboard the ISS. The SDTO experiments will both extend the results of the aircraft tests, and examine many of the practical aspects of component-level repair in a spacecraft environment.

CLEAR will also perform analysis on samples from an actual, unplanned repair performed by an astronaut aboard the ISS. These efforts, collectively, should serve to raise the maturity of component-level electronics repair as a repair strategy to a TRL level of 6 , and pave the way towards implementation of this strategy in future, longduration space missions.

\section{Acknowledgments}

The authors wish to thank Gary Latta and Andrew Ganster of the Naval Surface Warfare Center, Eric Anderson of Zin Technologies, and Gary Gorecki of NASA Glenn Research Center for their assistance in this effort. This work is funded by the Supportability Project in NASA's Exploration Technology Development Program Office (Barmac Taleghani, Program Manager, NASA Langley Research Center).

\section{References}

\footnotetext{
${ }^{1}$ Pettegrew, R.D., Easton, J., Struk, P.M., Anderson, E., “In-Flight Manual Electronics Repair for Deep Space Missions”, IEEEAC Paper \#1208, Submitted for IEEE Conference, March, 2007; also to be a NASA TM, spring, 2007.

${ }^{2}$ Columbia Accident Investigation Board, “Columbia Accident Investigation Board Report”[online report], Vol. 1, August, 2003 URL: http://www. caib.nasa.gov/news/report/volume1/default.html.

${ }^{3}$ Pettegrew, R.D., Struk, P.M., Watson, J.K., and Haylett, D.R., "Experimental Methods in Reduced-Gravity Soldering Research,” NASA TM-2002-211993, Dec. 2002.

${ }^{4}$ Pettegrew, R.D., Struk, P.M., Watson, J.K., Haylett, D.R., and Downs, R..S., "Gravitational Effects on Solder Joints,” Welding Journal, Vol. 82, No. 10, Oct. 2003, pp. 44-48.

${ }^{5}$ Struk, P.M., Pettegrew, R.D., Downs, R.S., and Watson, J.K., “The Effects of an Unsteady Reduced Gravity Environment on the Soldering Process,” AIAA Paper 2004-1311 and NASA TM-2004-212946, Jan. 2004.
} 
${ }^{6}$ Struk, P.M., Pettegrew, R.D., Downs, R.S., and Watson, J.K., “The Influence of Gravity on Joint Shape for Through-Hole Soldering,” AIAA Paper 2005-0541 and NASA TM-2005-213589, Jan. 2005.

${ }^{7}$ Watson, J.K., Struk, P.M., Pettegrew, R.D., and Downs, R.S., "Experimental Investigation of Solder Joint Defect Formation and Mitigation in Reduced-Gravity Environments”, AIAA Journal of Spacecraft and Rockets (to be published).

${ }^{8}$ Carlberg, T. and Liljendahl, M., "Soldering Under Microgravity," Proceedings of the 4th European Symposium on Materials Sciences under Microgravity, ESA SP-191, 1983, pp. 337 - 342.

${ }^{9}$ Grugel, R.N., Cotton, L.J., Segre, P.N., Ogle, J.A., Funkhouser, G., Parris, F., Murphy, L., Dillies, D., Hua, F., and Anilkumar, A.V., "The In-Space Soldering Investigation (ISSI): Melting and Solidification Experiments Aboard the International Space Station”, AIAA Paper 2006-521, Jan. 2006.

${ }^{10}$ Lekan, J., Neumann,E.S., and Sotos, R.G. Capabilities and Constraints of NASA's Ground-Based Reduced Gravity Facilities, NASA CP-10113. 1992.

${ }^{11}$ Johnson Space Center Reduced Gravity Program Office, “JSC Reduced Gravity Program User’s Guide”, Rev. A PCN 1, Document Number AOD 33899, August, 2005.

${ }^{12}$ NASA Technical Standard NASA-STD-8739.1, "Workmanship Standard for Staking and Conformal Coating of Printed Wiring Boards and Electronic Assemblies".

${ }^{13}$ Station Detail Test Objective website, URL: http://iss-www.jsc.nasa.gov/ss/issapt/mio/SDTO.html, Dec., 2006.

${ }^{14}$ Mankins, J.C., "Technology Readiness Levels: A White Paper”, internal document, URL: http://www.aa.washington.edu/courses/aa420/lectures/TRL_Definitions.pdf, Dec., 2006. 